\title{
Rett syndrome, classical and atypical: genealogical support for common origin
}

\author{
Hans Olof Åkesson, Bengt Hagberg, Jan Wahlström
}

\begin{abstract}
Aims of the study-By using genealogical methods in atypical females with Rett syndrome (RS) we looked for support for the assumption that atypical $R S$ cases are true variants of classical $R S$.

Subjects-We selected from the Swedish national RS series the "milder" RS cases, 10 years of age and older, fulfilling the criteria for the "forme fruste" (FF) type of RS. For 32 FF cases we were able to carry out complete genealogical analyses on 61 parental lines. The pedigrees contained details of about 3200 ancestors.
\end{abstract}

Common geographical origins-Eleven (34\%) of the FF females could be traced to a previously defined "Rett area" and no fewer than six females had their origin in the same homestead as another previously examined classical RS patient.

Ancestry-In four pedigrees, two each contained one FF and two classical RS and two each contained one FF and one classical RS, all 10 being descendants of the same four couples who lived several generations ago.

Consanguinity-Consanguinity in four grandparents $(6.6 \%$ (SD $3.2 \%)$ ) is probably a higher frequency than in the average Swedish population and supported our findings from a series of classical RS.

Transmission-The data indicate that transmission starts with a premutation that over generations can result in a full mutation giving rise to $R S$. Both the $X$ chromosomes and a pair of autosomes may be involved.

Institute of Clinical

Neuroscience,

Department of

Psychiatry,

Sahlgrenska University

Hospital, S-413 45

Göteborg, Sweden

H O Åkesson

Department of

Paediatrics, East

Hospital, Göteborg

University, Göteborg,

Sweden

B Hagberg

Division of Clinical Genetics, East

Hospital, Göteborg

University, Göteborg,

Sweden

J Wahlström

Correspondence to:

Professor Åkesson.

Received 12 March 1996 Revised version accepted for publication 14 May 1996 "forme fruste", as previously defined from Sweden, ${ }^{3}$ together with other neurologically mild forms, ${ }^{1}$ dominate, and represent roughly $85 \%$ or more of non-classical RS. ${ }^{2}$
By using genealogical methods on consecutively traced and examined cases of classical RS $(n=96)$ in two earlier studies, ${ }^{45}$ we have observed a statistically higher rate of consanguinity in the pedigrees examined, as well as a tendency to common origins. These observations suggest unusual genetic transmission.

Since the phenotypic expression varies, we thought it would be of interest to see if our genealogical observations could also be traced from a representative sample comprising only atypical forms of RS.

Aims of the study

By using the same genealogical method as formerly applied in classical RS, ${ }^{45}$ we examined the pedigrees of another Swedish sample comprising consecutively ascertained females but with atypical RS. Our hypothesis was that if a high rate of consanguinity and common geographical origins and ancestry were observed again, this would support the assumption that the atypical forms of RS are true variants of the nuclear group of RS and thus probably have a similar genetic background.

\section{Subjects and methods}

From the Swedish national RS series ${ }^{26}$ we selected the "milder" RS cases, 10 years of age and older, that fulfilled the original or later criteria for "forme fruste" (FF) types of RS. ${ }^{13}$ This included 34 RS-FF females, among whom five represented "preserved speech variants" and four represented "late regression" variants according to previously given clinical descriptions. ${ }^{3}$ All included were seen and the diagnosis confirmed by the child neurologist in the team $(\mathrm{BH})$.

In the main, this sample of 34 atypical RS females born in various parts of Sweden represented the more than $90 \%$ of atypical RS cases defined as $\mathrm{FF}^{3}$ with allied subgroups. ${ }^{1}$ It included one ambulant 17 year old girl who had shown developmental delay from earliest infancy, and who had convincingly also lost previously acquired skills. For our purposes the sample was reduced to 32 pedigrees, as two RS females had parents of foreign extraction on both sides. Another three maternal lines were unavailable for genealogical research: two were foreign and one included inaccurate maternal register data. Thus, complete genealogical analyses were carried out on 32 paternal and 29 maternal lines, or on 61 parental lines. The lines were traced as far back as possible, in most cases to $1720-1750$ or seven to 10 generations. The pedigrees contain details of about 3200 ancestors. 
Table 1 Common geographical origins of atypical and classical RS females

\begin{tabular}{lllllll}
\hline & \multicolumn{2}{l}{ Atypical $R S(n=32)$} & & \multicolumn{2}{l}{ Classical $(n=96)$} \\
\cline { 2 - 3 } Origin & No of pedigrees & $\%$ & & No of pedigrees & $\%$ \\
\hline Same Rett area & 11 & 34 & & 49 & 51 \\
Same homestead as another RS & $6(\times 2)$ & 19 & & $28(\times 2)$ & 29 \\
\hline
\end{tabular}

* For explanation see text.

\section{Results}

COMMON GEOGRAPHICAL ORIGINS

In two earlier Swedish series of classical RS, ${ }^{45}$ we noted that a high proportion of the oldest ancestors originated from a few small, sparsely populated areas that we have called "Rett areas". They consisted of nine areas located in all parts of Sweden. In six of them, pedigree clustering was confined to a single rural parish that had no more than 500 to 1000 inhabitants. The other three "Rett areas" contained equally few inhabitants and were as small as the six parishes but located on parish borders. To make certain we did not come across these "Rett areas" by chance, the definition in the first paper on classical RS females required the origin of at least three cases with RS in one area.

The same tendency was again observed among our series of RS-FF females. No fewer than $11(34 \%)$ of the pedigrees could be traced to one of these nine areas. Of these 11 pedigrees, five originated paternally and six maternally in a single "Rett area". In one pedigree the paternal ancestors had their roots in one "Rett area" and the maternal ancestors in another. In table 1, 11 (34\%) of the atypical cases are compared with 49 (51\%) of the classical females.

A remarkable previous finding among the 96 cases of classical RS was that in 28 instances two RS females came not only from the same small locality but even from the same homestead. By homestead we mean farm, household, or small group of dwellings. A similar observation was noticed in six pedigrees among the atypical RS females (table 1). Of particular interest is the finding that in all six instances the other RS female from the same homestead was of the classical type.

Thus, the same tendency regarding geographical origin was observed in the atypical group as in the two classical RS groups.

ANCESTRY: REMOTE COMMON COUPLES

In the previous series of classical RS females we showed in three cases that two of the females could be traced back to a single remote couple. We also found two instances, each of three Rett females, of descent from the same remote couple.

In the present study, when analysing FF female's ancestors, we again observed common couples that could be traced to around the late 18 th or early 19 th century. In two instances, two RS females were found to descend from such a single rather remote couple. In another two instances (one of which has recently been described $^{5}$ ), three girls originated from the same remote couple of ancestors.

In the present sample we had an aunt-niece pair that we have not included in this context.
They have previously been described by $\mathrm{us}^{57}$ and are descendants of a remote common couple. It is of interest to note, however, that the niece had classical RS while the aunt was of the non-classical type.

One of the RS-FF females was at first misclassified as belonging to the atypical group, but was later correctly classified by one of us $(\mathrm{BH})$ as belonging to the classical group according to the definition used. We found her, however, to originate from a remote couple, as had earlier been observed in the pedigree of one of the classical RS females.

Among the pedigrees explored of 129 females with RS we have therefore found that altogether $19 \mathrm{RS}$ females belonged to eight pedigrees. Three of the pedigrees contained three RS females, two of them including two classical and one non-classical RS cases. One comprised three classical RS females. A further observation was of five pairs of RS cases with a common remote single pair of ancestors; these five pairs consisted of three pairs of two classical RS and two pairs each of one classical and one non-classical or FF type of RS.

Four pedigrees thus contained a mixture of classical and non-classical RS females though they had a single pair of common ancestors several generations back. This finding speaks strongly in favour of a common genetic background for both typical and atypical RS females. In other words it could be said to strengthen the concept that FF females are true variants of the core group of classical RS cases.

CONSANGUINITY

None of our $32 \mathrm{RS}-\mathrm{FF}$ probands was born to first cousin couples, but quite a high frequency of cousin marriages was found among the grandparents on both sides. In fact, three grandfathers and one grandmother were offspring of cousins. The constellations vary, however. Of the grandfathers, one was born to first cousins once removed (also referred to as $11 / 2$ cousins), one was born to double second cousins, and the third was born to first cousins. The grandmother was born to second cousins. Thus, the inbreeding coefficients $(F)$ are quite different. In the first case $F=1 / 32$, in the second $F=1 / 32$, while in the third $F=1 / 16$. In the maternal line the grandmother was born to second cousins, which gives $F=1 / 64$.

We know that the rate of consanguineous marriage in Sweden has always been low; it has stayed quite constant at around $1 \%$ since 1850 , when such marriages were allowed without royal dispensation. ${ }^{458}$

When we pooled our two series of classical RS females, we found that $7.3 \%$ (SD $1.9 \%$ ) of the parents (13 out of 172 lines examined) were born to cousins. Assuming the true frequency of cousin marriages, at least of first cousin marriages, to be $1 \%$, our finding that $7.3 \%$ (SD $1.9 \%$ ) were offspring of cousins (confidence interval: 0.039-0.124) was statistically highly significant $(\mathrm{p}<0.001)$ (two tailed test).

Because of the variations in type of consanguineous relationships in the present series we 
found, owing to lack of accurate data in the normal population, no reason to try to test our observations statistically against expectations in the normal population.

\section{Discussion}

The main aim of the present study was to find out, by using genealogical methods, whether or not there is support for the assumption that atypical forms as defined are true variants of the nuclear group of RS. If this showed common geographical origins and ancestry as well as a raised rate of consanguinity among the grandparents, atypical and typical RS cases would probably have a common genetic background.

The answer must be that many, if not most, of the Rett variants studied are most likely descendants of the same RS family. The finding that the four kindreds with common ancestry contained both typical and atypical forms of RS speaks strongly in favour of a common background, as does the finding that $34 \%$ of the pedigrees examined had their roots in one of the earlier defined "Rett areas".

Around the beginning of the 19th century Sweden had about 1300 rural parishes with a total of approximately 2 million inhabitants and 200000 homesteads. There are thus considerable odds against the pure chance occurrence of (1) 49 of 96 classical RS females and 11 of 32 atypical RS females originating from the same nine areas or parishes, and (2) 28 pairs of classical RS females and six pairs of atypical RS females having ancestors in a common homestead. These facts are hard to ignore.

Concerning common ancestors, the striking convergence of family trees seen in the present study is further emphasised by the fact that a similar genealogical study, given the same historical and geographical conditions, covering 96 known cases of neurofibromatosis showed parental lines scattered over the country with no tendency to clustering in small areas. The ancestors showed completely random distribution in their geographical origins and no single common couple of remote ancestors was observed. ${ }^{9}$

As to the question of how far affected subjects breed "true to type", our data indicate that the same variability was displayed that one would expect to see in a normal cross section of patients with RS.

As to the rate of consanguineous marriages in the present series of atypical cases of RS, it was probably higher than that in the general population. Four consanguineous marriages among the grandparents of 61 lines explored $(6.3 \%$ (SD $3.2 \%)$ ) fit the previous results noted among our classical RS cases. Owing to the variations in the type of consanguineous relationship, we have no data indicating the expected frequency of such constellations in the normal population. A bold guess would be that the corresponding figure would be between 1 and $1.5 \%$, but as we cannot prove this there is no reason to try to test such a presumptive difference statistically.

The mode of inheritance of typical and atypical RS is still unknown. It might be of a kind not yet described. We believe that a premutation takes place first in an early generation and that a RS girl will carry the full mutation. The premutation could be carried over generations by several possible mechanisms, perhaps by amplification of trinucleotides, as known, for example, in the fragile $\mathrm{X}$ syndrome. Our data point to a two gene model. Based on theoretical considerations, such a model has been suggested by Bühler et al. ${ }^{10}$ Their hypothesis, which fits our genealogical data, involves one $\mathrm{X}$ linked and one autosomal mutation. They also calculated with the existence of both female and male carriers and suggested that one combination of mutations in the two genes is compatible with forme fruste or atypical Rett syndrome. In our presumptive carriers in the eight pedigrees with a remote single couple transmitting the premutation or premutations, it is worthy of note that our carriers often pass the mutation from father to son. Our data thus involve both male and female carriers and include atypical cases, as suggested by Bühler et al. ${ }^{10}$

The general conclusions to be drawn from our different genealogical studies on RS are that we have to take into account not only the influence of an X chromosomal defect but also the possibility of autosomal interactions.

This study was supported by grants to $\mathrm{H} \mathrm{O}$ Åkesson from the Royal Society of Arts and Sciences in Gothenburg, the SävstaRoyal Society of Arts and Sciences in Gothenburg, the SavstaNo 3921) and by a grant to B Hagberg from the W:6 Rett No 3921) and by
Research Support.

1 Hagberg B, Skjeldal OH. Rett variants: a suggested mode for inclusion criteria. Pediatr Neurol 1994;11:5-11.

2 Hagberg B. Rett syndrome: clinical pecularities and biological mysteries. Acta Paediatr 1995;84:971-6.

3 Hagberg B, Witt Engerström I. Rett syndrome: a suggested staging system for describing impairment profile with increasing age towards adolescence. Am $\mathcal{F}$ Med Genet 1986;24(suppl 1):47-59.

4 Åkesson HO, Hagberg B, Wahlström J, Witt Engerström I. Rett syndrome: a search for gene sources. Am $\mathcal{F} M e d$ Genet 1992;42:104-10.

5 Åkesson HO, Wahlström J, Witt Engerström I, Hagberg B Rett syndrome: potential gene sources - phenotypical variability. Clin Genet 1995;48:169-72

6 Hagberg B, Witt Engerström I. The Swedish series of females with Rett syndrome 1960-1992. In: Hagberg B, ed. Rett syndrome - clinical and biological aspects. Clinics in developmental medicine, No 127. London: Mac Keith Press, 1993:21-6.

7 Anvret M, Wahlström J, Skogsberg P, Hagberg B. Segregation analysis of the $\mathrm{X}$-chromosome in a family with Rett syndrome in two generations. Am $f$ Med Genet 1990; 37:31-5.

8 Alström CH. First-cousin marriages in Sweden 1750-1844 and a study of the population movement in some Swedish subpopulations from the genetic-statistical viewpoint. Acta Genet (Basel) 1958;8:295-369.

9 Samuelsson B, Åkesson HO. Neurofibromatosis in Gothenburg, Sweden. Genetic analyses. Neurofibromatosis 1989 2:107-15.

10 Bühler EM, Malik NJ, Altran M. Another model for inheritance of Rett syndrome. Am $\mathcal{F}$ Med Genet 1990;36:126-31. 